\title{
Multilingual Conversational Systems to Drive the Collection of PROs and Integration into Clinical Workflow
}

\author{
DANIEL HARI \\ Faculty of Electrical \\ Engineering and \\ Computer Science \\ University of Maribor, \\ Maribor, SLOVENIA
}

\author{
VALENTINO ŠAFRAN \\ Faculty of Electrical \\ Engineering and \\ Computer Science \\ University of Maribor, \\ Maribor, SLOVENIA
}

\author{
UMUT ARIOZ \\ Faculty of Electrical \\ Engineering and \\ Computer Science \\ University of Maribor, \\ Maribor, SLOVENIA
}

\author{
IZIDOR MLAKAR \\ Faculty of Electrical \\ Engineering and \\ Computer Science \\ University of Maribor, \\ Maribor, SLOVENIA
}

\author{
MATEJ ROJC \\ Faculty of Electrical \\ Engineering and \\ Computer Science \\ University of Maribor, \\ Maribor, SLOVENIA
}

\author{
GAZIHAN ALANKUS \\ Emoda Software, \\ İzmir, TURKEY
}

\author{
RAFAEL PEREZ LUNA \\ Dedalus Company, \\ Sevilla Area, SPAIN
}

\begin{abstract}
Collection of patient-reported outcomes (PROs) remotely and their usage in the clinical workflow provide an improvement on both patient's quality of life and cancer care. However, adoption of collecting PROs into the clinical workflow is rare, and existing works still have a lot of issues providing a holistic approach. This paper offers enhancements in the process of collecting PROs by utilization of conversational systems that still provide quite a new but promising way to collect PROs remotely with spoken interaction. Our proposed system provides an interoperability with Fast Healthcare Interoperability Resources (FHIR) server by using a multimodal sensing network (MSN) prepared for the project PERSIST. We introduce components of multimodality while collecting PROs with the help of the mHealth App and Open Health Connect (OHC) platform. As a result, chatbots and 3D embodied conversational agents (ECA) were prepared to interact with the cancer patients in 5 different languages. The intercommunication was provided by MSN and the integration of cancer patients' PROs into clinical workflow was satisfied. This study was part of a Horizon 2020 project and a preparation phase for clinical trials with cancer patients and clinicians.
\end{abstract}

Keywords - chatbot, embodied conversational agent, patient reported outcomes, FHIR, multimodal sensing network.

Received: March 2, 2021. Revised: July 9, 2021. Accepted: July 30, 2021. Published: August 10, 2021.

\section{Introduction}

Patient-reported outcomes (PROs) are an interesting subject in healthcare because of their usage for improving patients' experience, quality of life, and participation of the patient into clinical workflow [1]. To get the highest contribution from PROs, the most suitable PROs should be selected for improving healthcare. At this point, another important point appears: how to collect data from patients, how to integrate them into clinical workflow, and how to display them to the physicians. Besides those points, things like facilitating the use of PROs for the patients, the cost and time for answering, and implementation of PROs should be kept in mind. Otherwise, some problems such as rejection of further use of provided PROs by patients may occur.

The first usage of patient outcomes was proposed to improve healthcare in 1988 [2]. The Medical Outcomes Study presented concepts of patient data collection, comparisons between different areas of Patient-Reported Outcomes (PROs), and ideas for the development of PROs [3]. During these times, patient outcomes were collected mostly face-to-face, using forms, or with minimal use of technology in the form of phone calls. With the emergence of communication technologies, the use of SMS, the internet, and e-mail is emphasized to collect PROs due to the accessibility and rapid expansion of mobile phones [4]. In many studies, the usage of electronic questionnaire apps on smartphones and their feasibility, acceptability, and efficiency was shown $[5,6]$. Nowadays, there is an attempt to use new technologies to help improve healthcare and quality of life (QoL) of the patients by giving access to PROs via smartphones and using machine learning and other branches of artificial intelligence [7,8].

In recent years, conversational systems are shown as the most promising technologies to provide remote data gathering electronic PROs from patients. Conversational systems include Chatbots, Speech Dialog Systems (SDS), and Embodied Conversational Agent (ECA) technologies. These systems started with ELIZA, a chatbot that emulated a psychotherapist [9]. Nowadays, these kinds of technologies are used in many domains, ranging from banking to game environments.

Chatbots are systems able to converse and interact with human users using spoken, written, and visual languages [10]. In the context of healthcare, chatbots are intended to provide patients with personalized health and therapy information, relevant products and services, to connect them with health care providers as well as suggest diagnoses and recommended treatments based on patient symptoms and reports. Having properties like cost-effectiveness, multilingual communication, and $24 / 7$ availability make chatbots useful for patients with medical concerns outside of their doctor's operating hours. Also, studies reported that patients perceive chatbots as safer interaction partners than human physicians and are willing to disclose more medical information and report more symptoms to them [11].

Another type of conversational system are Embodied Conversational Agents (ECA). One of the earliest definitions of ECA is "more or less autonomous and intelligent software 
entities with an embodiment used to communicate with the user" [12]. The main three components of ECAs are user interfaces for communication with ECA; computer modelling structure for reacting the ECA emphatically; and embodiment (visual representation) for communication with users. Embodiments can be designed as virtual human characters [13], animals [14], or robots [15]. Although ECAs are more realistic and have multimodal input capacity, ECAs need more complex infrastructures, more development and implementation times, and more multidisciplinary expertise than chatbots. The choices should be done according to the concept of the project to find an optimal configuration. The main areas of ECAs in healthcare are the treatment of mood disorders, anxiety, psychotic disorders, autism, and substance use disorders [16]. In a review of Kramer [17] about the design and evaluation of the ECAs for healthcare, ECAs proved a promising tool for persuasive communication in healthcare. And, in another review study [15], technological and clinical possibilities of less complex ECAs were investigated and shown as a solution for routine applications in the means of rapid development, testing, and application. The design features of ECAs for healthcare were investigated by Stal [18] and found that the agents' speech and/or textual output and its facial and gaze expressions as the most used features for ECAs.

To provide a realistic environment to patients, agents' speech is the most important part of the ECAs. This can be provided by Speech Synthesis or Text-to-Speech (TTS) technology. With the usage of deep learning algorithms, the synthesized waveforms can sound very natural and almost indistinguishable from how a human would speak. Such TTS models can be used in cases like when an interactive virtual assistant responds to a patient while performing a questionnaire.

To benefit from PROs via chatbots or ECAs, that information should be integrated into clinical workflow to achieve improvement in patients' quality of life and to provide decision support to physicians. The integration of patient data still poses a problem in healthcare. Fast Healthcare Interoperability Resources (FHIR) is the promising solution for integration and interoperability for healthcare. FHIR enables the creation, editing, deletion, and exchange of definitions of medical sources for specific profiles such as Patient, Observation, Questionnaire, and more than 140 other resources [19]. HAPI FHIR, an open-source implementation of the FHIR in Java, allows converting between FHIR sources and other application data, to access external server resources, to allow external applications to access or edit application data, and to introduce a fully functional FHIR server where the necessary applications can be developed.

This study has been performed within a Horizon 2020 project (PERSIST, https://projectpersist.com/). In this study, we proposed a complete system for collecting PROs remotely via both chatbots and ECAs and integrating the PROs into the clinical workflow by using FHIR. The FHIR server is located at the open health connect (OHC) platform. All traffic will be managed by a multimodal sensing network composed of different microservices like TTS, an ECA, a chatbot, and automatic speech recognition (ASR). This study will be the baseline for the clinical trials with cancer patients which will be our next study. In section II, related works and the differences of our study will be explained; the methodology and the results will be given in sections III and IV, respectively. In section V, our main contributions will be discussed and the paper will end with the conclusion section.

\section{Related work}

Up to now, most of the studies focus on the development of chatbots/ECAs or on defining the interoperability issues for the clinical workflow. In our study, we proposed a holistic approach for the integration of PROs into clinical workflow.

In recent studies [20,21], active engagement was satisfied by PROs for cancer patients. Although they reported effective mechanism and satisfaction in perioperative care for patient participation, the integration into clinical workflow and usage as part of routine care were not mentioned. However, in our study, we achieved the integration of PROs into clinical workflow in a very effective way for both patients and clinicians.

After improvements for natural language processing, studies showed the capacity of chatbots for complex tasks like booking tickets, fetching the result from API and therefore acting as customer service agents $[22,23]$. In our study, we additionally use a Rasa engine for a chatbot with the FHIR system to collect the PROs from patients and to present it both on patient and on clinician mobile applications. Another advantage of our study is to be able to know how our patients will collaborate with chatbot and how chatbots affect the result of the study, since we strive for a chatbot-patient conversation that is as natural as possible.

Sayeed et al. [24] describe an approach to create a patientcentered health system that is based on FHIR standard and patients/clinicians' applications that can make requests and reports of HL7 FHIR resources. Their baseline workflow is the collection of PGHD and forming of FHIR resources; but in our study, we combine FHIR resources with microservices of MSN as a novel approach.

Previous ECA studies for healthcare mainly focused on physical activity [25-27], nutrition [28,29], stress [30], blood glucose monitoring [31], and sun protection [32]. Our study will be the first ECA for collecting PROs.

Kramer [17] reviewed 20 ECA studies to compare their functionalities and appearances. For the appearance of ECA, most of the studies used similar virtual human characters like middle-aged African American women. In our study, we used middle-aged European women and men as virtual human characters. For the communication style, only one ECA was reported that uses both speech and text like our proposed system. Also, while most of the ECAs gave permission to users to choose a single response among the predefined list, we provided a storyline conversation to the patients. Among the reviewed 20 studies, only 3 studies gave information about the design of the embodiment. The rest of the studies provided brief or no information about their ECAs. Another important difference of our study is multilingualism. For the reviewed ECAs, there is no information about language. In our study, we 
will provide multilingualism by providing a speech in English, Slovenian, French, Russian, and Spanish (these are the languages of the PERSIST project).

\section{Methods}

The main focus of our study is a multimodal sensing network (MSN) and usage with microservices. The system (Fig. 1) can be divided into three main units: mHealth applications, MSN, and OHC FHIR server.

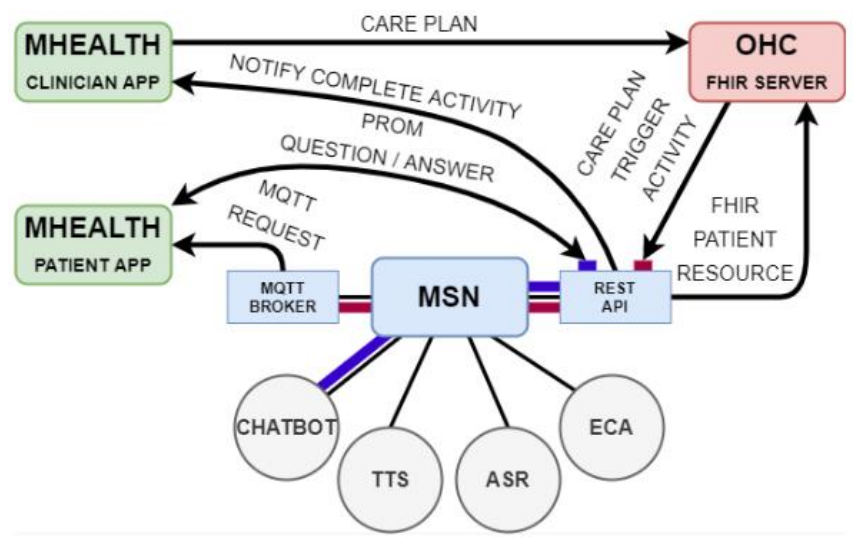

Fig. 1. Overall system - clinician and patient mHealth App, OHC FHIR server, and MSN.

\section{1. mHealth App}

In the study, patients and clinicians have separate mHealth applications (Fig. 2). The patient mHealth application are mainly used for data gathering and monitoring the trends; the clinician mHealth application is mainly used for monitoring the patients and specifying the care plans for patients. Both mobile applications were developed by the company Emoda.

The patient application has the functionalities such as mood selection, diary recordings, reading of selected articles by clinicians, answering the questionnaires, receiving messages from the clinician, and clinician appointment scheduling as the main functionalities of the application. For the clinician application, clinicians have options to see all the patients' lists and their clinical details. They are able to create a new patient record and edit or delete an already existing one. Also, clinicians can create appointments, see the calendar, receive alerts for specific patients, and send/receive messages from patients.

The mHealth App can use both synchronous and asynchronous protocols. While synchronous REST protocol is used for communication with OHC and MSN REST OpenAPI (Swagger) endpoints, asynchronous MQTT protocol is used for receiving notifications.

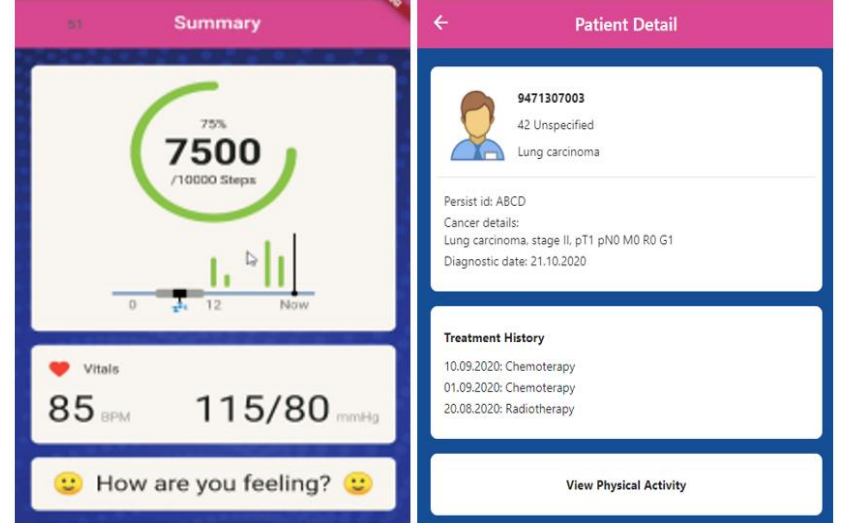

Fig. 2. Patient (left) and clinician (right) mHealth App first version.

\subsection{OHC FHIR server}

The Open Health Connect (OHC) platform is the complete integration and streaming platform for large-scale distributed Environments provided by the company Dedalus. OHC enables all the interfaces to be connected to and make decisions across disparate data sources in real-time. The OHC Digital Health Platform comprises a set of components as depicted in the conceptual/logical architectures. The $\mathrm{OHC}$ solution is flexible and can be deployed on-premise (private data center) or cloud in environments like Azure or AWS. OHC provides the latest version of HAPI FHIR R4 [33].

\subsection{Multimodal Sensing Network}

The main building blocks in MSN consist of Apache Camel and ActiveMQ Artemis for providing interconnectivity among the blocks, and Apache Kafka for providing a multimodality component. Apache Camel can act as a router by having the ability to convert synchronous messages to asynchronous and vice versa. Apache Camel can also run as a Spring Boot application that provides REST API endpoints for HTTP requests. ActiveMQ Artemis is the MQTT broker which is an intermediate between $\mathrm{OHC}$ and mHealth App. Here, mHealth App is working as an MQTT client subscribed to ActiveMQ Artemis. Microservices are interconnected using Kafka topics and HTTP APIs. For Kafka services, asynchronous communication is used where predefined topics for each language are supported and synchronous communication is used for Rasa chatbot that uses HTTP REST requests over Camel REST endpoints API.

The Microservice Architecture (MA) is used for MSN as a series of small services or separate processes. Four microservices are used in this study. The first microservice is Speech synthesis, Text-to-speech (TTS) microservice. It mainly generates audio files with given transcriptions for ECA that communicate with the patients. Two types of TTS architecture are used. The first TTS architecture is developed for real-time or close to real-time systems by combination of two neural network models: a modified Tacotron 2 model and a flow-based neural network model - WaveGlow. The second TTS architecture is developed for non-real-time system use with better quality output by the combination of two neural 
network models: a modified Tacotron 2 model and a Wavenet model.

ASR, the second microservice, is feeding text transcription to the Rasa chatbot where language is determined and processed. ASR systems can be built using a number of approaches depending on input data type, intermediate representation, model type, and output post-processing. In this study, ASR focuses on end-to-end Connectionist Temporal Classification (CtC) based models which take speech samples and transcripts without any additional information (like the original DeepSpeech model).

The third microservice are the ECAs, virtual characters that serve as communication partners to patients and offer a component of non-verbal interaction in addition to verbal. Male and female ECAs were developed for our study. Our platform consists of the three cooperative frameworks/platforms: conversational analysis platform, embodied virtual agent (EVA) framework, and EVA realization framework.

As a fourth microservice, the Rasa Chatbot is used in our system like an API, where Rasa is representing the engine of the chatbot. The chatbot is running on a Linux server and is programmed in python and YAML language. Our API implements 18 questionnaires in six PERSIST project languages. Those questionnaires are representing guidelines for the clinical studies in the PERSIST project. For storing the data, Rasa API uses SQLite database which is possible with a function called SQLTrackerStore in the Rasa chatbot. POST and GET requests are used to store the information like patients' answers, questionnaires, and all events that are triggered in a specific conversation.

\section{Results}

The main results of our study are the workflow of the system proposed with a focus on Questionnaires and ECAs (EVA for female version and ADAM for male version) for spoken interaction.

The detailed process of the system is explained in the UML diagram (Fig. 3 and Fig. 4). The process starts with the creation of a care plan for the patient by the clinician that is stored in $\mathrm{OHC}$ as FHIR Resource. Then, OHC is triggering the activity for the patient mHealth App by communicating with the appropriate MSN REST API endpoint. The notification about the triggered activity is sent through MSN's MQTT broker to the patient mHealth App in JSON data format. The patient can see five types of notifications: (1) request to fill the Questionnaire, (2) request to provide information about the mood, (3) request to record a diary, (4) notification of a received message from the clinician, and (5) some other notifications.

After the patient finishes the assigned Questionnaire, MSN creates an FHIR resource which is sent to both the OHC FHIR server and the clinician to be notified about the completion of the activity.

In the proposed structure, Rasa provides a way to start a message for determination about starting time, an end message for determination about ending time, and questions with corresponding answers for sending another questionnaire to patients. When patients want to start a Questionnaire service, they need to run a command to start a questionnaire over our REST API, and then a request to start a session is sent.

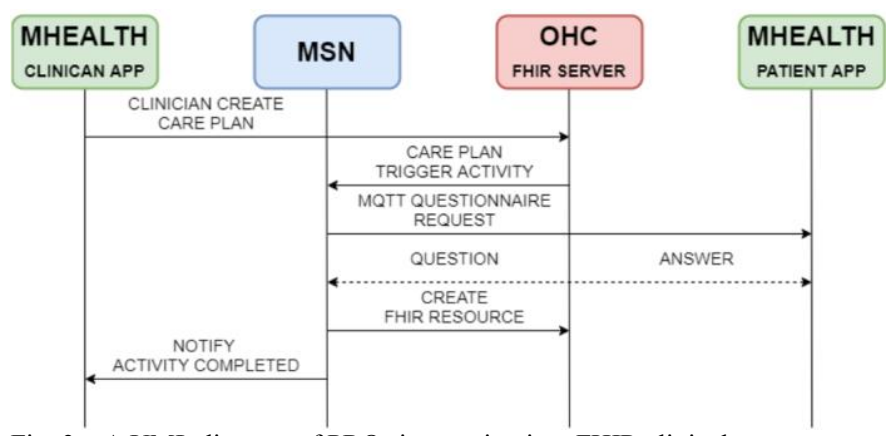

Fig. 3. A UML diagram of PROs integration into FHIR clinical resources.

After that, the user can select given answers or write to the chatbot by using the functionalities of HTTP POST requests and HTTP GET requests of REST API. There are many events (session started, pause, resume, slot, restart) that can trigger conversations and can contribute to better conversation performance. With GET requests, the history of patients' conversations with the Rasa chatbot can be seen by clinicians to store and review reports for improved patient diagnosing. Questionnaires are available in 5 different languages of PERSIST project: Slovenian, English, Russian, French, and Spanish.

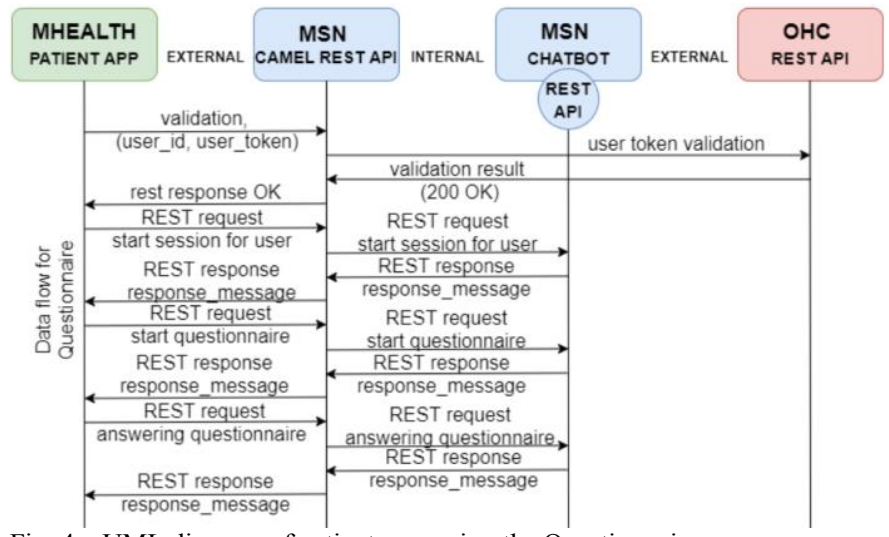

Fig. 4. UML diagram of patient answering the Questionnaire

' $E v a$ ' is represented as a middle-aged European woman and 'Adam' as a middle-aged European man (Fig. 5). Our ECAs are bringing spoken interaction to this system by converting unannotated texts into co-verbal behaviour description by the behaviour generation model (BGM) proposed in [34].

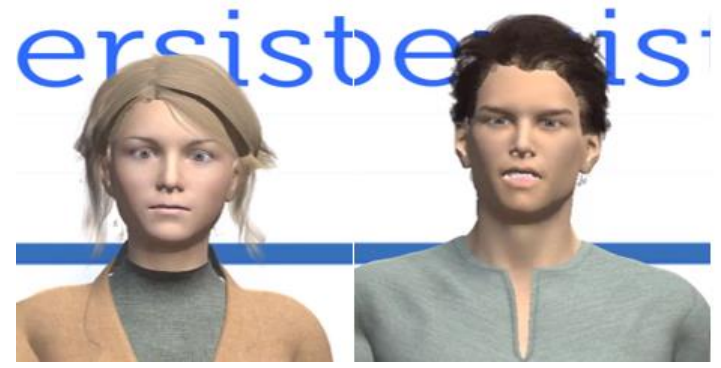

Fig. 5. 'Eva' and 'Adam' embodied conversational agents 
Non-verbal elements are associated with speech. Unannotated texts are given as multimodal output which offers a spoken communication channel as well as a synchronized visual communication channel. BGM is synchronizing the verbal and non-verbal elements for our ECAs to act more naturally, more human-like. The patient mHealth App will include our ECAs in the future versions which should contribute to better patient participation.

\section{Discussion}

The aim of our study is to provide a holistic approach for remotely gathering patient PROs in a more natural and storyline format via multilingual conversational systems and to integrate into clinical workflow for clinician use for better healthcare and increased quality of life of the cancer patients. Spoken interaction via chatbot or ECAs (Eva and Adam) and integration of the patient data into clinical workflow are the main contributions of our study.

A significant barrier across the integration of the PGHD into clinical workflow is the inflexibility of the medical systems. Although there are some studies for this obstacle, there is still a gap for multimodal data integration into EHR records. However, many of the installed systems are unable to adapt to any update in the healthcare system. One of the solutions is to develop a FHIR infrastructure and to have widespread usage of the FHIR compatible systems.

Accessing the data, importing PROs into the treatment report, managing with lots of data, and interpreting the PROs are some of the reported barriers for using PROs in the clinic [35]. All mentioned barriers can be removed with integration into clinical workflow and better visualization by FHIR which is the trending opportunity for overcoming interoperability problems [36].

This study proposed a complete system that includes patient/clinician mobile applications, OHC FHIR server, and multimodal sensing network. The patient/clinician mobile applications are designed according to the security concerns and clinical trial requirements. For using the main functionalities of the mHealth App, users (patients and clinicians) have to complete a few steps. The first step starts with user registration and password change after the first registration process. Afterward, the consents are shown based on the native browser element provided by OHC. Once these steps are successfully passed, the smart band connection and OHC connection are checked as a final step. Then, users can reach the main functionalities of the applications. When users are signed in, the mHealth app starts to collect data from the smart band and continues to work in the background.

Interoperability among the components is provided by the OHC FHIR server in the study. OHC provides the framework and set of tools for the integration, ingestion, storage, indexing, and surfacing of patient information. It is an innovative, open digital integration hub with the proven ability to deliver the speed, scale, and flexibility needed to securely gain value through the integration of health systems. By consolidating data in a standardized format (FHIR) from across a broad range of systems of record, $\mathrm{OHC}$ enables innovation through near-realtime access to longitudinal patient records.

Multimodal sensing network was designed as microservice infrastructure. In this infrastructure, the building blocks are each divided into their own service and are scaled so that the services are distributed among the servers and can be replicated as needed. MSN was designed as four components: TTS, ASR, the EVA framework, and the chatbot.

For inference of the TTS engine, we set up the NVIDIA Triton Inference server which simplifies the deployment of AI models at scale in production. All TTS models will be deployed in the NVIDIA Triton server repository. ASR engines are running on the same workstation as client applications in python language. Their input will be the text files transferred from the Kafka network. TTS engine clients for all languages will process accepted text files. As output, clients will forward the created audio files with input transcriptions. This will be used in the second step for Forced Alignment, producing timealigned phonemes in the audio signal. Then, this is sent into the Kafka framework via specific topics, while Kafka broker delivers TTS outputs to corresponding consumers (e.g. animation engine).

ASR was developed by focusing on end-to-end Connectionist Temporal Classification (CtC) based models. $\mathrm{CtC}$ allows finding an alignment between audio and text.

In this study, two ECAs were implemented. The conversational analysis platform is used for analysing how linguistic and paralinguistic features interplay with embodiments during complex, spontaneous, and multiparty interactions. The EVA framework is capable of capturing various contexts in the "data" and providing the basis to analytically investigate various multidimensional correlations among co-verbal behaviour features. The role of the EVA realization framework is to transform the co-verbal descriptions contained in EVA events into articulated movement generated by the expressive virtual entity, e.g., to apply the EVA-Script language onto the articulated 3D model EVA in the form of animated movement [37]. Also, multilingual properties of our ECAs differentiates it from other similar ECAs.

The Chatbot API is using PREMs and PROMs to see patients' health status and patients' perceptions of their experience whilst receiving treatment All sent and received data is in JSON format due to easy usage for transmitting data in web applications, and better representation and understanding. That is important because doctors should see the history of the report for specific patients in an understandable form to give more accurate decisions. For conversations between the Rasa Chatbot API and patients, it is important to configure story.yaml which contains the flow of the conversation or the order of the intents to be executed, which depends on the patients responses [38].

\section{Conclusion}

Collection of PROs in an easier way and integrating patient data into clinical workflow are among the main factors for improving the quality of life of cancer patients. Although there are some studies for collection of PGHD and integration of data 
into clinical workflow, our proposed system presents a complete solution that provides both in a multilingual fashion.

With this study, we tried to show the applicability of the usage of microservices with a multimodal sensing network as an interface among patients, clinician and the FHIR server. All proposed components were implemented successfully. The interconnections among all parts of the system and conversational systems (chatbot and ECAs) were developed as expected and published at our lab's website (https://dsplab.feri.um.si/en/dsplab/).

In this study, we provided an infrastructure with relevant questionnaires for our conversational systems. In future, we will have a flexible dialog that will take place according to the given patient's answers and other patient's metrics as a clinical trial.

\section{Acknowledgement}

This study is part of the project 'PERSIST: Patient-centered survivorship care plan after cancer treatment' that has received funding from the European Union's Horizon 2020 research and innovation programme (GA No. 823748).

\section{References}

[1] „What are Clinician-Reported Outcomes (ClinROs)?“, National Health Council, September 26, 2019.

[2] P. M. Ellwood, „Outcomes Management“, N. Engl. J. Med., year 318, n. 23 , p. $1549-1556,1988$.

[3] A. R. Tarlov, J. E. Ware Jr, S. Greenfield, E. C. Nelson, E. Perrin, in M. Zubkoff, ,The Medical Outcomes Study: An Application of Methods for

[4] E. Bielli, F. Carminati, S. La Capra, M. Lina, C. Brunelli, in M. Tamburini, „A Wireless Health Outcomes Monitoring System: development and field testing with cancer patients using mobile phones", BMC Med. Inform. Decis. Mak., year 4, n. 1, p. 7, June 2004.

[5] C. Tran, A. Dicker, B. Leiby, E. Gressen, N. Williams, in H. Jim, „Utilizing Digital Health to Collect Electronic Patient-Reported Outcomes in Prostate Cancer: Single-Arm Pilot Trial“", J. Med. Internet Res., year 22, n. 3, p. e12689, 2020.

[6] A. A. Wright idr., ,The HOPE Pilot Study: Harnessing Patient-Reported Outcomes and Biometric Data to Enhance Cancer Care", JCO Clin. Cancer Inform., n. 2, p. 1-12, 2018.

[7] P. Rajguru, S. Ryan, E. McLaurin, D. Wirta, in J. Grieco, „A novel method for collecting patient reported outcomes (PROs): developing and validating electronic PROs on a mobile smartphone platform", Invest. Ophthalmol. Vis. Sci., year 61, n. 7, p. 110-110, 2020

[8] L. S. E. van Egdom, A. Pusic, C. Verhoef, J. A. Hazelzet, in L. B. Koppert, "Machine learning with PROs in breast cancer surgery; caution:

[9] Weizenbaum, J. (1966). A computer program for the study of natural language communication between man and machine. Communications of the ACM, 9(1), pp. 36-45.

[10] B. Shawar and E. Atwell, 'Chatbots: Are they Really Useful?', LDV Forum, vol. 22, pp. 29-49, 2007.

[11] A. Palanica, P. Flaschner, A. Thommandram, M. Li, and Y. Fossat, 'Physicians' Perceptions of Chatbots in Health Care: Cross-Sectional Web-Based Survey', J. Med. Internet Res., 21, no. 4, p. e12887, 2019.

[12] Isbister K, Doyle P. The blind men and the elephant revisited evaluating interdisciplinary ECA research. In: Ruttkay Z, Pelachaud C, editors. From brows to trust evaluating embodied conversational agents. Dordrecht, Netherlands: Springer; 2004:3-26.

[13] Bickmore T, Gruber A, Picard R. Establishing the computer-patient working alliance in automated health behavior change interventions. Patient Educ Couns 2005 Oct;59(1):21-30.

[14] Klaassen R, Bul KCM, op den Akker R, van der Burg GJ, Kato PM, di Bitonto $P$. Design and evaluation of a pervasive coaching and gamification platform for young diabetes patients. Sensors (Basel) 2018;18(2):pii: E402.

[15] Provoost S, Lau HM, Ruwaard J, Riper H. Embodied Conversational Agents in Clinical Psychology: A Scoping Review. J Med Internet Res. 2017 May 9;19(5):e151.

[16] Brinkman WP. Virtual Health Agents for Behavior Change: Research Perspectives and Directions. In: Proceedings of the Workshop on Graphical and Robotic Embodied Agents for Therapeutic Systems. 2016 Presented at: GREATS'16; September 20, 2016.
[17] Kramer, L. L., Ter Stal, S., Mulder, B. C., de Vet, E., \& van Velsen, L. (2020). Developing Embodied Conversational Agents for Coaching People in a Healthy Lifestyle: Scoping Review. Journal of medical Internet research, 22(2), e14058.

[18] Silke ter Stal, Lean Leonie Kramer, Monique Tabak, Harm op den Akker, Hermie Hermens, Design Features of Embodied Conversational Agents in eHealth: a Literature Review, International Journal of HumanComputer Studies, Volume 138, 2020.

[19] Resourcelist - FHIR v4.0.1. http://hl7.org/fhir/resourcelist.html (retrieved April 01, 2021).

[20] Kneuertz, P. J., Jagadesh, N., Perkins, A., Fitzgerald, M., Moffatt-Bruce, S. D., Merritt, R. E., \& D'Souza, D. M. (2020). Improving patient engagement, adherence, and satisfaction in lung cancer surgery with implementation of a mobile device platform for patient reported implementation of a mobile device platform for pat
outcomes. Journal of thoracic disease, 12(11), 6883-6891

[21] Girgis A, Durcinoska I, Levesque JV, Gerges M, Sandell T, Arnold A, Delaney GP, The PROMPT-Care Program Group eHealth System for Collecting and Utilizing Patient Reported Outcome Measures for Personalized Treatment and Care (PROMPT-Care) Among Cancer Patients: Mixed Methods Approach to Evaluate Feasibility and Acceptability J Med Internet Res 2017;19(10): 3330.

[22] Rakesh Kumar Sharma and National Informatic Center, 'An Analytical Study and Review of open source Chatbot framework, Rasa', Int. J. Eng. Res., vol. V9, no. 06, p. IJERTV9IS060723, 2020.

[23] M. Joshi and R. K. Sharma, 'An Analytical Study and Review of open Source Chatbot framework, RASA', Int.J.Eng. Res. Technol., 9-6, 2020.

[24] R. Sayeed, D. Gottlieb, in K. D. Mandl, ,SMART Markers: collecting patient-generated health data as a standardized property of health

[25] Friederichs S, Bolman C, Oenema A, Guyaux J, Lechner L. Motivational interviewing in a web-based physical activity intervention with an avatar: randomized controlled trial. J Med Internet Res 2014 Feb 13;16(2):e48.

[26] Bickmore TW, Caruso L, Clough-Gorr K, Heeren T. 'It's just like you talk to a friend' relational agents for older adults. Interact Comput
2005;17(6):711-735.

[27] Ellis T, Latham NK, DeAngelis TR, Thomas CA, Saint-Hilaire M, Bickmore TW. Feasibility of a virtual exercise coach to promote walking in community-dwelling persons with Parkinson disease. Am J Phys Med Rehabil 2013;92(6):472-81; quiz 482.

[28] Blanson Henkemans OA, van der Boog PJ, Lindenberg J, van der Mast CA, Neerincx MA, Zwetsloot-Schonk BJ. An online lifestyle diary with a persuasive computer assistant providing feedback on self-management. Technol Health Care 2009;17(3):253-267.

[29] Bickmore TW, Schulman D, Sidner C. Automated interventions for multiple health behaviors using conversational agents. Patient Educ Couns 2013;92(2):142-148.

[30] Gardiner PM, McCue KD, Negash LM, Cheng T, White LF, YinusaNyahkoon L, et al. Engaging women with an embodied conversational agent to deliver mindfulness and lifestyle recommendations: a feasibility randomized control trial. Patient Educ Couns 2017;100(9):1720-1729.

[31] Klaassen R, Bul KCM, op den Akker R, van der Burg GJ, Kato PM, di Bitonto $P$. Design and evaluation of a pervasive coaching and gamification platform for young diabetes patients. Sensors (Basel) 2018;18(2):pii: E402.

[32] Sillice MA, Morokoff PJ, Ferszt G, Bickmore T, Bock BC, Lantini R, et al. Using relational agents to promote exercise and sun protection: assessment of participants' experiences with two interventions. J Med Internet Res 2018;20(2):e48.

[33] Versions - FHIR v4.0.1. https://www.h17.org/fhir/versions.html (retrieved April 01, 2021).

[34] Rojc M, Mlakar I, Kačič Z. The TTS-driven affective embodied conversational agent EVA, based on a novel conversational-behavior generation algorithm. Eng. App. of AI. 2017;57:80-104.

[35] Rotenstein LS, Agarwal A, O'Neil K, et al. Implementing patientreported outcome surveys as part of routine care: lessons from an academic radiation oncology department. J Am Med Inform Assoc. 2017;24:964-968.

[36] Heather S. L. Jim, Aasha I. Hoogland, Naomi C. Brownstein, Anna Barata, Adam P. Dicker, Hans Knoop, Brian D. Gonzalez, Randa Perkins, Dana Rollison, Scott M. Gilbert, Ronica Nanda, Anders Berglund, Ross Mitchell, Peter A. S. Johnstone. Innovations in research and clinical care using patient- generated health data. CA: A Cancer Journal for
Clinicians, 2020.

[37] Matej Rojc, Zdravko Kačič and Izidor Mlakar (March 30th 2018). Advanced Content and Interface Personalization through Conversational Behavior and Affective Embodied Conversational Agents, Artificial Intelligence - Emerging Trends and Applications, Marco Antonio Aceves-Fernandez, IntechOpen.

[38] A. Singh, K. Ramasubramanian, and S. Shivam, 'Introduction to Microsoft Bot, RASA, and Google Dialogflow', in Building an Enterprise Chatbot: Work with Protected Enterprise Data Using Open Source Frameworks, Eds. Berkeley, CA: Apress, 2019, pp. 281-302.

\section{Creative Commons Attribution License 4.0 (Attribution 4.0 International, CC BY 4.0)}

This article is published under the terms of the Creative Commons Attribution License 4.0

https://creativecommons.org/licenses/by/4.0/deed.en_US 